\title{
APAWSAN: Actor Positioning for Aerial Wireless Sensor and Actor Networks
}

\author{
Mustafa İlhan Akbaş and Damla Turgut \\ Department of Electrical Engineering and Computer Science \\ University of Central Florida \\ Email: \{miakbas,turgut $\} @$ eecs.ucf.edu
}

\begin{abstract}
The node mobility is a natural element of many wireless sensor and actor network (WSAN) applications. Recent advances in the development of small unmanned aerial vehicles (UAVs) with built in sensors made it possible to deploy aerial sensor and actor networks. An aerial network composed of small UAVs enables high quality observation for events while reducing the number of personnel and the risk for the operators. In order to have an effective data collection, the positioning of actors plays a critical role in aerial WSANs.

In this paper we propose an actor positioning strategy for aerial WSANs considering the scenario of toxic plume observation after a volcanic eruption, which is one of the emerging applications of aerial UAV networks. Measuring the composition of volcanic plumes allows the computation of volcanogenic fluxes and provides insights into volatile degassing mechanisms. The actors in the proposed approach use a lightweight and distributed algorithm to form a self organizing network around the central UAV, which has the role of the sink in the WSAN. Our algorithm makes use of the Valence Shell Electron Pair (VSEPR) theory of chemistry, which is based on the correlation between molecular geometry and the number of atoms in a molecule. The performance of the proposed practical positioning algorithm is presented through extensive simulations.
\end{abstract}

\section{INTRODUCTION}

Wireless sensor and actor networks (WSANs) [1] consist of a large number of sensor nodes, which have limited capabilities, and a smaller number of actors, which process the collected information and react according to the data. The node mobility is a natural element of many WSAN applications and it permits the network to be autonomous during deployment. The recent advances in development of small unmanned aerial vehicles (UAVs) with built in sensors made it possible to deploy autonomous aerial sensor and actor networks, which do not rely on human intervention.

There are examples of mini UAVs with various built-in sensors. Some of these devices have the size of a fulmar and they are inexpensive compared to fully equipped research aircrafts. Therefore, UAV systems are cost effective and attractive solutions for surveillance. There are emerging industry concepts for using UAVs with remote control or as an autonomous system in critical missions. One of the emerging applications is the development of an aerial network composed of small UAVs, which enables high quality observation for events such as toxic plume behavior or storm dynamics and provides a unique three dimensional system for environmental monitoring. In such a system, additional UAVs acting as mo- bile base stations can improve network performance measures such as energy consumption and traffic load balancing.

In the context of this paper, we consider the scenario of a volcanic eruption such as the eruptions of the volcano Eyjafjallajökull at Eyjafjöll in Iceland in the Spring of 2010. The erupting lava of Eyjafjallajökull injected a cloud of ash into the Jet Stream and caused the highest level of air travel disruption since the World War II. When the large quantities of dry volcanic ash lying on the ground are combined with sulphurous gases emitted and surface winds, an "ash mist" was lifted up and the visibility was reduced considerably. The close-up observation of the volcano, the plume and the surroundings became impossible. In the application scenario considered in this paper, a UAV system with built-in sensors is used to collect data from the volcanic plume as shown in Fig. 1.

The UAV system in the scenario performs measurements in the plume and at locations close to the volcanic vents. It is either impossible or it has a high risk potential to conduct these tests by personnel. Measuring the composition of volcanic plumes allows the computation of volcanogenic fluxes and provides insights into volatile degassing mechanisms. Examples of the observations executed by the UAV system include $\mathrm{SO}_{2}$ determinations and IR measurements. The utilization of UAV systems in this application reduces the number of personnel, the risk for the operators, intervention time while increasing the quality in observation, investigation and the response.

The UAV system considered in an application scenario consists of small UAVs with the role of sensor nodes in a WSAN and larger actor UAVs. The particular problem investigated within this concept is the dynamic positioning of the actors in three dimensional space, which is critical for the effective data collection. In recent years, there has been an increasing interest in applications of sensor networks in three dimensional space such as space exploration, airborne and underwater surveillance, oceanic studies, storm tracking and so on. However most of the literature on dynamic node positioning strategies is limited to two-dimensional space and the well-known strategies designed for two dimensions become NP-Hard in three dimensional space. The optimization strategies for node positioning in 3-D setups are important and the existing solutions are only preliminary.

In this paper we propose APAWSAN, an actor positioning strategy for aerial WSANs. In APAWSAN, the actors use a 


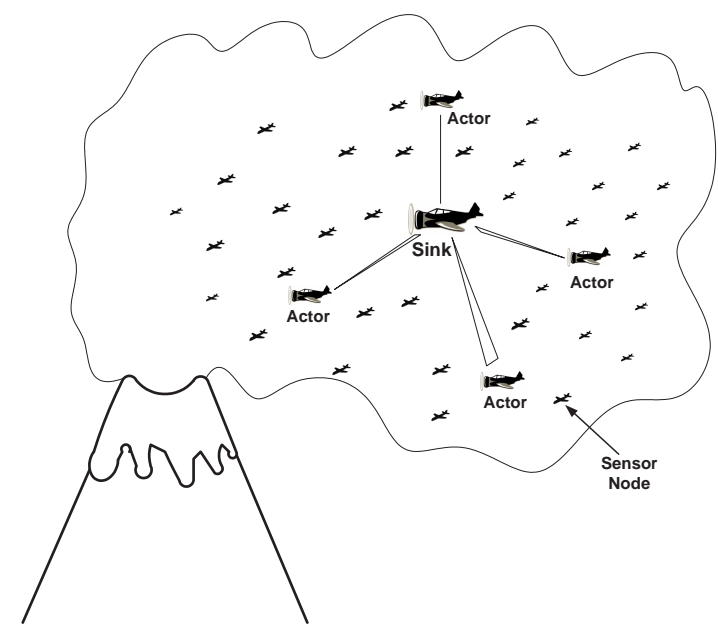

Figure 1. Volcano eruption application scenario.

lightweight and distributed algorithm based on the Valence Shell Electron Pair Repulsion (VSEPR) theory to form a self organizing actor network. VSEPR theory is originally used in chemistry for the prediction of the peripheral atom alignments around a central atom. This concept is adopted in APAWSAN to align actor UAVs around the UAV acting as the sink role.

The rest of the paper is organized as follows. Section II summarizes the related work. We provide a detailed description for our approach in Section III. We show the simulation results in Section IV and finally conclude in Section V.

\section{RELATED WORK}

The literature on 3-D wireless sensor networking mostly focus on coverage problems. In the conventional 2-D scenarios, a sensor coverage is generally modeled as a circle and the maximal coverage problem is mapped to a circle packing formulation which has a polynomial time solution. This problem turns into the sphere packing problem in three dimensions. Given the high complexity of the sphere packing problem for 3-D coverage, Alam and Haas [2] argue that space filling polyhedrons would be more suitable and try to fill the 3-D application space with the least number of polyhedrons in order to provide maximal coverage. Ravelomanana [3] studies the properties of the network topologies that result from random deployment of nodes in a 3-D region of interest to provide theoretical bounds that can help in preliminary design and feasibility studies of 3-D WSNs. The author derives conditions for the node transmission range $r$ required for achieving a degree of connectivity $d$, where every node has at least $d$ neighbors. Pompili et al. [4] uses Ravelomanana's bounds to validate the effectiveness of their 3-D random underwater node deployment scheme. Zhou et al. [5] present two algorithms for discovering boundary nodes and constructing boundary surfaces in 3-D wireless networks. Bai et al. [6] designed and proved the optimality of one and two connectivity patterns under any value of the ratio of communication range over sensing range, among regular lattice deployment patterns. The authors also introduced three and four connectivity patterns and investigated the evolutions among all of the proposed lowconnectivity patterns. Slab Routing by Chiang and Peng [7] adapts 2-D geographic face routing techniques to 3-D space by dynamically creating a space partition and executing face routing over the planar projected graph of nodes contained within.

There are aerial sensor network implementations for various applications concerning the measure of air pollution and weather variables. Only just a few experiments for volcanic gas sampling exists in the literature, while the autonomous aerial system by Astuti et al. [8] has a very similar application scenario with APAWSAN, In their system, there is no network but a single UAV, which performs aerial surveillance of volcanic areas and to analyze the composition of gases inside volcanic plumes. The SensorFly system [9] by Purohit and Zhang is a mobile-controlled flying sensor network that monitors changes in a dangerous environment such as an earthquake or fire. SensorFly uses a flying miniature sensor with a weight of $30 \mathrm{~g}$ and low mass production cost around \$100. Elston and Frew [10] [11] presents a hierarchical control architecture similar to APAWSAN with a mothership, which acts as a distributed database and daughtership micro air vehicles, which use vector field tracking. Autonomous Flying Robot MARVIN (Multipurpose aerial robot vehicles with intelligent navigation) project [12] uses robots with the ability to coordinate with each other to complete required tasks. SensorFlock by Allred et al. [13] is an airborne WSN composed of bird-sized micro aerial vehicles (MAVs), with a focus on the design of the MAVs and received signal strength indication (RSSI). Their WSN is composed of hundreds of inexpensive, semiautonomous, and cooperating airborne vehicles making observations and relaying data over a wireless communication mesh network.

APAWSAN makes use of the VSEPR theory of chemistry, which is based on the idea of a correlation between molecular geometry and the number of valence electrons around a central atom. This concept was first presented by Sidgwick and Powell [14]. Gillespie and Nyholm [15] refined it later and built the elaborate VSEPR theory, which states that the maximum repulsion of the electron pairs or atoms defines the geometric optimum positions of peripheral atoms or alone electron pairs that maximizes the distance between these entities.

\section{APAWSAN}

\section{A. System model}

There are three operation modes for UAVs in current technology [16]. The first operation mode is external piloting, in which the vehicle is controled by an operator using the line of sight. The second mode is internal piloting using a ground station with an on board camera. The last mode is the autonomous flight. The first two modes are not applicable for our application scenario. The line of sight is almost impossible to have in a volcan eruption since the plume is not transparent and personnel may not be located in a safe position to view the whole flight path. Moreover, the conditions change frequently, which makes autonomous flight necessary for our scenario. 
Autonomous flight on the other hand has some disadvantages, most of which are due to the fact that the operators cannot closely monitor the automated mission planning software. These issues may cause lower situational awareness and reduces the ability to correct errors in the system. Therefore the communication among the actors must be simple, but yet effective. Considering these conditions, APAWSAN forms a hierarchically structured WSAN of $N$ nodes, consisting of a set, $S$, of small UAVs, which have built-in sensor nodes and a set, $A$, of bigger and more powerful UAVs with actor nodes. There is also a sink node which is located on the largest sized UAV with the extended capabilities so that it is not affected by the expected or unprecedented environmental conditions.

The network among the actors and the sink is defined as the backbone of the network. The network backbone is maintained by periodical packet exchange between direct neighbors. The sink is a direct neighbor to each actor in the network and serves as the control center for the positioning of the nodes during the flight. Hence these roles form the hierarchical structure in the network. The sink is at the highest level in this hierarchy and its position is used by the positioning algorithm at actors to determine their positions. There are not predetermined positions relative to the sink for small UAVs carrying the sensors. They collect information in the plume and follow the same main flight path with the sink and the actors, but with a varying distance to the sink to collect measurements in the plume. Additionally, the small UAVs carrying the sensors are affected by the environmental conditions more than the actor and the sink UAVs. Therefore there are perturbations in the flight paths of these small UAVs, which requires a dynamic mechanism for the actor affiliation of the sensor nodes.

The affiliation of sensor nodes to the actors is executed similar to SOFROP [17]. The sensor nodes donot follow any predetermined configuration for initial affiliations. Each sensor node keeps only a "weight" value for each actor it is affiliated with, which represents the minimum number of hops required to reach the actor from that sensor node. The sensor nodes initially take random weight values between 0 and $k-1$ while each actor node is assigned to a constant weight $k$. The only data available for a sensor node $s$, are the direct neighbors $N e i g h(s)$ and their corresponding weights $w\left(N e i g h\left(s_{i}\right)\right)$. Hence, the sensor nodes maintain and update only local information.

The sensor nodes and actors are assumed to have transmission radii $r_{s}$ and $r_{a}$, respectively, with spherical transmission ranges, where $r_{s}<r_{a}$ due to better computation and communication capabilities of the actors. The sensor nodes affiliate with the closest actors and an actor uses $r_{s}$, when communicating with the affiliated sensor nodes. The actors use their full transmission range, $r_{a}$, only when communicating with another actor or with the sink.

For communication between two nodes, a bidirectional connection must be established. A UAV $U_{1}$ must be in the transmission range of the sensor on the neighboring UAV $U_{2}$, i.e $d\left(U_{1}, U_{2}\right) \leq r_{s}$ for communication. Each sensor node $(s)$ keeps a list $N e i g h(s)$ of 1-hop neighbors and every node can communicate only with these nodes, no multi-hop control communication is needed. The communication links may fail or disappear from the network caused by various reasons $\mathrm{s}$ uch as obstacles or characteristics of the plume.

\section{B. "VSEPR theory" based approach}

The VSEPR model, which is a natural extension of the electron pair model, has become the most successful and widely used model for the prediction of geometries of closedshell molecules. Within a molecule, the Laplacian of the electronic charge density exhibits extrema in the valence shell of the central atom. These extrema indicate the presence of localized concentrations of electronic charge. The local charge concentrations duplicate in number, location and size for the spatially localized electron pairs considered in the VSEPR model. In other words, the spherical surface on which the electron pairs are assumed to be localized in the VSEPR model is identified with the sphere of maximum charge concentration in the valence-shell charge concentration (VSCC) of the central atom and the localized pairs of electrons are identified with the local maxima on this sphere of maximum charge concentration. Hence the Laplacian of the charge density provides the physical basis for the VSEPR model.

The electron pairs in the valence shell of the central atom are arranged because of the repulsion between them. For two or three surrounding valence shell electron pairs, these arrangements can be predicted by using the points on a sphere model. In this model, there is a given number of points each representing a valence-shell electron pair and these pairs are confined to the surface of a sphere. However, for other cases such as the trigonal bipyramidal structure with equal bond lengths, the optimum geometry can not be predicted by the same method. Instead it is a trigonal bipyramid with axial rather than equatorial bonds. Therefore, an elaborate method is required for the prediction of the geometries in VSEPR model.

When using the VSEPR theory to find the geometrical structure of a molecule, the "AXE method" of electron counting is used. $A, X$ and $E$ represent the three important parameters of this method. $A$ is the number of central atoms, which is one for the fundamental VSEPR theory. $X$ is the number of sigma bonds between the central atom and the surrounding atoms. $X$ can also be considered as the number of atoms around the central atom, since multiple covalent bonds are counted as one when finding $X . E$ is the number of lone electron pairs surrounding the central atom. The sum of $X$ and $E$, known as the steric number, is also associated with the total number of hybridized orbitals used by valence bond theory. Based on the steric number and distribution of $X$ 's and $E$ 's, VSEPR theory makes the predictions in Fig. 2 to Fig. 5.

In APAWSAN, UAVs are used to build a self-sustaining, self-configuring dynamic network architecture. There are only a few applications in the literature with multiple UAV types forming a heterogenous aerial system, none of which includes more than a certain number of devices. Most of the implemented volcanic gas sampling systems use only one 
UAV. Therefore, the number of actors in APAWSAN doesn't exceed eight and there is one sink for data collection and aggregation. Sink is located in the center of the WSAN and it provides a coordination center with 1-hop distance to all other actors. This structure of actor positions makes the application scenario highly applicable for the atom positioning concept in VSEPR theory. VSEPR theory is applied to actor positioning in APAWSAN as follows:

- The peripheral atoms in VSEPR theory are mapped to the actors and the central atom is mapped to the sink. The possible actor positions for different number of actors are found. The found positions are converted into positions with respect to the sink in order to be used during flight.

- An designed algorithm allows the actors to position during the flight and for the transitions from one geometry to the other. Hence both for increasing and decreasing number of actors, there is a self-organizing system of UAVs handling sudden failures or temporary communication loss.

- Lone electron pairs are more repulsive than bound atoms and they occupy more space than atoms. This concept of VSEPR theory is adopted for the cases when there is a need to avoid positions in a particular part of the flight path, for instance when there are obstacles.

\section{Formulation of VSEPR geometries}

The locations of actors in the geometries formed using the VSEPR model must be identified according to a reference point. Therefore the position of the sink $p_{S}$ is taken as the reference origin in $X Y Z$ coordinate system during the flight and all other positions are calculated with respect to the origin. Additionally, the location options for actors must be formulated in a way such that the transition between geometries can be done with a lightweight algorithm at each actor node since the main aim is to have a self-organizing network of actors. The locations in possible geometries are formulated in this section to simplify the positioning algorithm and the transitions among the possible geometries during the flight.

The UAV system flying in the plume of the volcano has actors with similar features and "lone electron pair" in the "AXE method", which is used to implement VSEPR theory, doesn't correspond to an element of the system in a regular flight scenario. Therefore the possible actor positions are calculated according to the VSEPR thoery without any lone electron pairs, i.e $E$ is zero. The geometrical structures with lone electron pairs are used as an extension of the protocol. When the lone electron pair concept is used for extension, the actor positions calculated without lone-pairs are used as the basis to determine the actor positions.

When there is a single actor, it takes a position with a predefined distance of $r$ to the sink. This not considered as a geometry formed by the actors in APAWSAN because of its simplicity. However the communication between the sink and the actor described in system model, and therefore $r$, is still

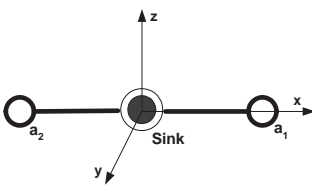

Figure 2. Linear geometry

important in the collection of the measurement data for the single actor.

The network of UAVs has a flight plan and all UAVs fly together as system according to this plan. Consequently, the main direction that the sink headed on a time instant forms the $x$-axis. The $y$-axis is perpendicular to $x$-axis and parallel to the earth's surface. Then $z$-axis is perpendicular to the surface that $x$ and $y$ axes form.

When there are two actors, the actors and the sink are arranged during the flight with an expected connection angle of $180^{\circ}$. This geometrical arrangement is called "Linear" geometry (see Fig. 2). The positons of the actors in Linear geometry are as follows:

$p_{a_{1}}(x, y, z)=(r, 0,0)$

$p_{a_{2}}(x, y, z)=(-r, 0,0)$

The molecular geometry model with a single atom at the center and three peripheral atoms at the corners of a triangle all in one plane is named as "Trigonal planar". This is the geometrical model used in APAWSAN when there are three actors around the sink. Fig. 3 (a) shows the Trigonal planar orientation of UAVs, in which all three actors are in identical distances to the sink with connection angles of $120^{\circ}$, given as follows:

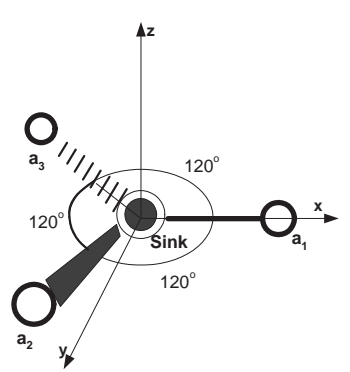

(a) Trigonal planar geometry.

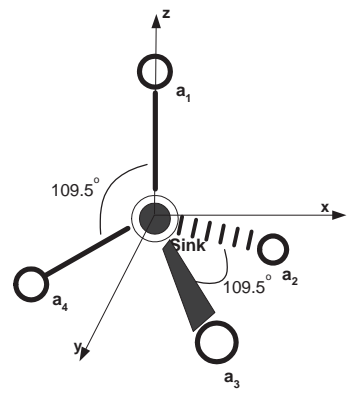

(b) Tetrahedral geometry.
Figure 3. Geometries formed by three and four actors.

$p_{a_{1}}(x, y, z)=(r, 0,0)$

$p_{a_{2}}(x, y, z)=\left(-r \cdot \sin \left(30^{\circ}\right), r \cdot \sin \left(60^{\circ}\right), 0\right)$

$p_{a_{3}}(x, y, z)=\left(-r \cdot \sin \left(30^{\circ}\right),-r \cdot \sin \left(60^{\circ}\right), 0\right)$

When there are four peripheral actor UAVs, the sink is located at the center with four substituents that are located at the corners of a tetrahedron as shown in Fig. 3 (b). This geometry is called Tetrahedral and the connection angles are 


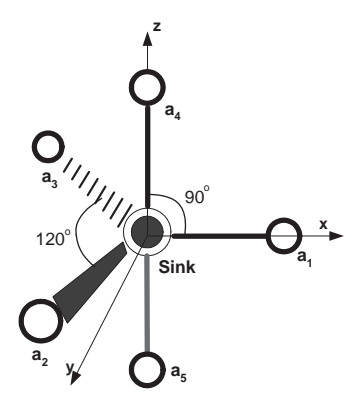

(a) Trigonal bipyramid geometry.

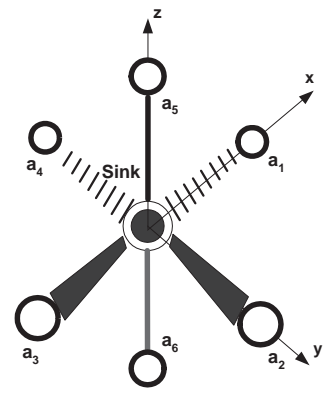

(b) Octahedral geometry.
Figure 4. Geometries formed by five and six actors.

$\cos ^{-1}(-1 / 3) \approx 109.5$ when all four actors are located at the positions calculated according to tetrahedral geometry.

$p_{a_{1}}(x, y, z)=(0,0, r)$

$p_{a_{2}}(x, y, z)=(-r . a,-r . b, r \cdot \cos (109.5))$

$p_{a_{3}}(x, y, z)=\left(-r \cdot \sin \left(109.5^{\circ}\right), 0, r \cdot \cos (109.5)\right.$

$p_{a_{4}}(x, y, z)=(-r . a, r . b, r \cdot \cos (109.5))$

where $a=\sin \left(109 \cdot 5^{\circ}\right) \cdot \sin \left(30^{\circ}\right) b=\sin \left(109 \cdot 5^{\circ}\right) \cdot \cos \left(30^{\circ}\right)$

There is no geometrical arrangement for one sink and five actors which can result in five equally sized connection angles in three dimensions. When there are five actors surrounding the sink, they take positions during the flight with non-identical connection angles relative to the sink. As shown in Fig. 4 (a), three actors are positioned on the $y=0$ plane with connection angles of $120^{\circ}$ whereas the other two actors take positions on $y$-axis with angles of $90^{\circ}$ to the $y=0$ plane. Hence the geometry formed with each actor at a corner of this geometry is a Trigonal bipyramid.

$p_{a_{1}}(x, y, z)=(r, 0,0)$

$p_{a_{2}}(x, y, z)=\left(-r \cdot \sin \left(30^{\circ}\right), r \cdot \sin \left(60^{\circ}\right), 0\right)$

$p_{a_{3}}(x, y, z)=\left(-r \cdot \sin \left(30^{\circ}\right),-r \cdot \sin \left(60^{\circ}\right), 0\right)$

$p_{a_{4}}(x, y, z)=(0,0, r)$

$p_{a_{5}}(x, y, z)=(0,0,-r)$

When there are six actors, they are arranged around the sink symmetrically, defining the vertices of an octahedron as shown in Fig. 4 (b). The octahedron has eight faces as its prefix implies and the final geometry is an Octahedral with an actor at each corner.

$p_{a_{1}}(x, y, z)=(r, 0,0)$

$p_{a_{2}}(x, y, z)=(0, r, 0)$

$p_{a_{3}}(x, y, z)=(-r, 0,0)$

$p_{a_{4}}(x, y, z)=(0,-r, 0)$

$p_{a_{5}}(x, y, z)=(0,0, r)$

$p_{a_{6}}(x, y, z)=(0,0,-r)$

The pentagonal bipyramid (or dipyramid) is a molecular geometry with one atom at the center with seven ligands at the corners of a pentagonal dipyramid. Therefore it is the geometry used when there are seven actors, as shown in Fig. 5 (a). Other seven coordinate geometry possibilities include the mono-capped octahedron and mono-capped trigonal prism. However pentagonal bipyramid is chosen for APAWSAN considering its suitability for transition between geometries in cases such as the loss or an addition of an actor. Similar to trigonal bipyramid, the connection angles are not identical in this geometry.

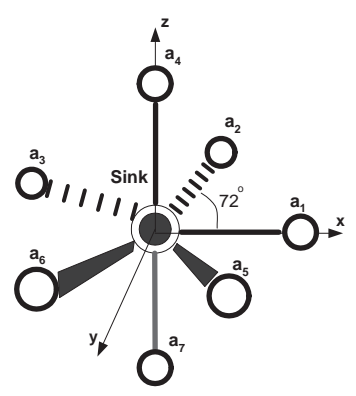

(a) Pentagonal bipyramid geometry.

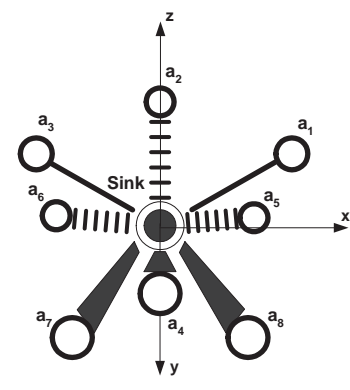

(b) Square antiprismatic geometry.
Figure 5. Geometries formed by seven and eight actors.

$$
\begin{aligned}
& p_{a_{1}}(x, y, z)=(r, 0,0) \\
& p_{a_{2}}(x, y, z)=\left(r \cdot \cos 72^{\circ}, r \cdot \sin 72^{\circ}, 0\right) \\
& p_{a_{3}}(x, y, z)=\left(-r \cdot \cos 36^{\circ}, r \cdot \sin 36^{\circ}, 0\right) \\
& p_{a_{4}}(x, y, z)=(0,0, r) \\
& p_{a_{5}}(x, y, z)=\left(r \cdot \cos 72^{\circ},-r \cdot \sin 72^{\circ}, 0\right) \\
& p_{a_{6}}(x, y, z)=\left(-r \cdot \cos 36^{\circ},-r \cdot \sin 36^{\circ}, 0\right) \\
& p_{a_{7}}(x, y, z)=(0,0,-r)
\end{aligned}
$$

According to the VSEPR theory, the square antiprism is the favored geometry when eight atoms surround a central atom. A square anti-prism corresponds the shape when eight points are distributed on the surface of a sphere with the aim of maximising the distance between each pair. It is also called as an anticube and it is the geometry used in APAWSAN when there are eight actors as shown in Fig. 5 (b).

$$
\begin{aligned}
& p_{a_{1}}(x, y, z)=(r . a \sqrt{2} / 2,0, r . h / 2) \\
& p_{a_{2}}(x, y, z)=(0, r . a \sqrt{2} / 2, r . h / 2) \\
& p_{a_{3}}(x, y, z)=(-r . a \sqrt{2} / 2,0, r . h / 2) \\
& p_{a_{4}}(x, y, z)=(0,-r . a \sqrt{2} / 2, r . h / 2) \\
& p_{a_{5}}(x, y, z)=(r . a, r . a,-r . h / 2) \\
& p_{a_{6}}(x, y, z)=(-r . a, r . a,-r . h / 2) \\
& p_{a_{7}}(x, y, z)=(-r . a,-r . a,-r . h / 2) \\
& p_{a_{8}}(x, y, z)=(\text { r.a, }- \text { r.a, }- \text { r.h } / 2)
\end{aligned}
$$

where $a$ and $h$ are constants used in pentagonal bipyramid geometry to simplify the transitions. $h / 2 \approx 0.5237$ represents the positive and negative $z$ values for the planes that the actors are located at and $a \approx 1.2156$.

\section{Dynamic positioning}

The positioning alternatives of the actors are given with respect to the sink and to each other. However UAVs are not 
stationary and our dynamic application scenario includes various requirements and challenges because of its differentiating features. The main challenges and their projections for the system are given as follows:

- There is not an operation center or a remote control option. The UAVs operate in an extreme conditions and they must operate without a human control requirement from the start of the flight to the end.

- The system is continuously in motion with a certain speed. Therefore the positioning must be flexible so that small changes in positions relative to the sink does not affect the general geometry. This is critical for data collection.

- The changes and the maintanence of the actor positions must be accomplished through local communication only. The actors communicate only with their direct neighbors. The communication among sensor nodes and between actors and sensor nodes is also local only.

- The actors must be able to reorganize in case of a loss or an addition of an actor. Due to the differentiating features of the scenario, the actors can be damaged or lost during the operation. The transitions between the probable geometries must be well defined and executable with local communication.

The coordination among the actors for positioning at optimum locations is carried out in ASPAWAN with the described challenges taken into consideration. All UAVs have a predetermined flight plan in the application scenario for the autonomous operation. However each actor UAV needs to coordinate with others to obtain and maintain the best location in the system for volcanic gas sampling objectives. Making use of VSEPR theory, a lightweight algorithm is designed for the determination of the actor positions during the flight in the aerial WSAN in volcano eruption scenario.

The positioning of an actor in the aerial network is given in Algorithm 1. The most important parameter for an actor to decide on its location is the number of actors $(n)$. Since the sink is in 1-hop distance to each actor, it always keeps the information about the $n$ and it updates actors with the periodical message exchange. Hence it informs all actors when there is a change in $n$.

In Algorithm 1, the common properties of calculated positions of actors in the specifc geometries or their relations are used. For example, the planes on which multiple actors are positioned in three dimensional space is one of these properties. Another one is the angle between the position vectors of two neigboring actors. Both of these are main characteristics of the geometries and they are used in the actor positioning algorithm. The actors decide on their positions during the flight using these parameters and the communication with their 1hop neighbors. Each actor flies with the same distance, $r$, to the sink.

All actor position options according to Algorithm 1 are on $z=0$ plane when $n$ is smaller than four. Therefore, depending on their initial positions, the actors exchange packets with their

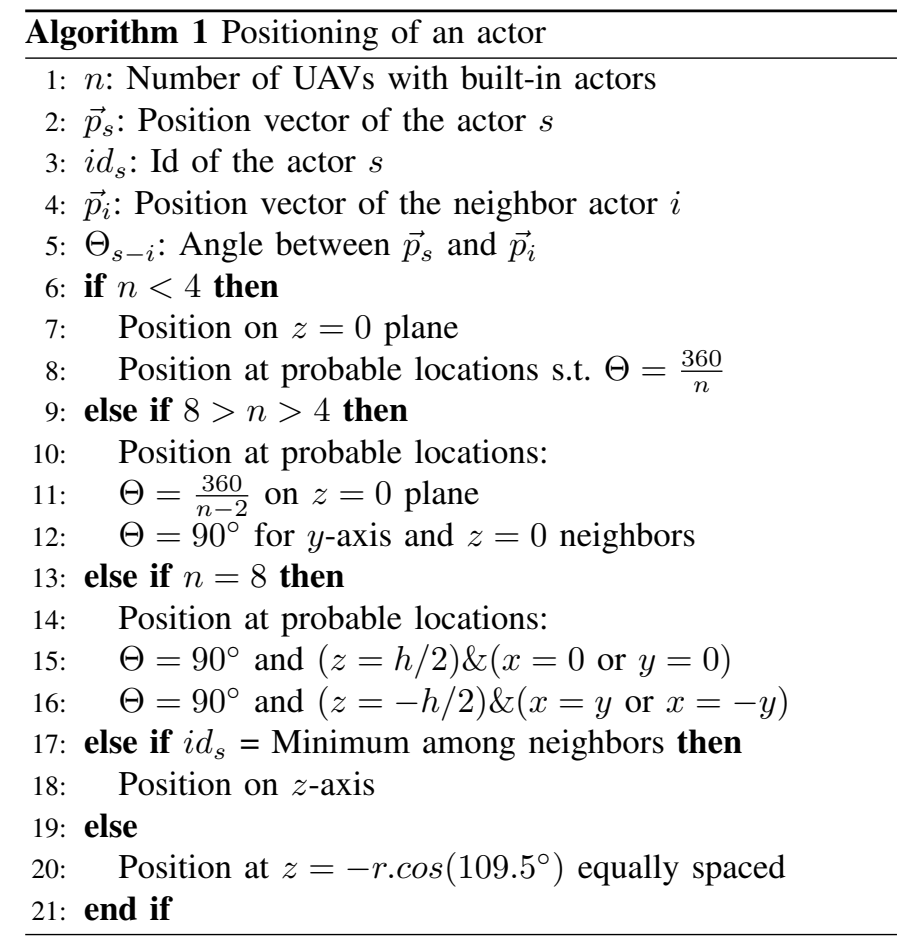

1-hop neighbors and get positions on $z=0$ plane, satisfying the condition $\Theta=\frac{360}{n}$.

When $n$ is equal to four, the geometry is perfectly simetrical. Therefore, in a system with distance measurement capabilities and a rather stable or expectable conditions, the actors can form this geometrical structure simply by satisfying the conditions for the distances among them. However, our application scenario includes a system operating in extreme conditions and one of the main goals is having a lightweight and fast algorithm for actor positioning. Therefore, the actor closest to $z=r$ plane takes $(0,0, r)$ position and the other nodes take their positions according to Algorithm 1. All actors are located with equal distances to each other in this geometry. Thus, the actors use only a $z$ value and local communication to take their positions.

When $n$ is greater than four and smaller than eight, there are two positions on $z$ axis and the other actor positions are on $z=0$ axis with the condition $\Theta=\frac{360}{n-2}$. As the number of actors increases, it is easier for an actor to position itself in the correct spot since the communication possibility among actors increases with decreasing distances. The geometry formed by the actors is a square antiprismatic when $n$ is eight. The characteristic features of this geometry is used to define the actor positions to be used in our algorithm. In square antiprismatic, four of the eight actors are the plane $y=h / 2$ and the other four are on the plane $y=-h / 2$, both of which are parallel to $X Y$ plane. The actors position themselves using only two $z$ values and the information they gathered by periodical packet exchange with direct neighbors. 


\section{Simulation STUdy}

\section{A. Simulation environment}

The simulation study is conducted in the OPNET modeler [18]. IEEE 802.11 forms the MAC layer for sensor nodes and actors. The transmission radius of a sensor node is 40 meters. Each node includes a 20 packet-size queue and a data rate of 10 packets per second.

There are 30 sensor nodes, one sink and two to eight actors in the simulation scenarios. For each simulation scenario, 30 runs are executed with these parameters and the parameters specific to the executed scenario.

The communication graph is built according to the system model specified in Section III. The communication links that sensor nodes form may fail or disappear from the network. The system of UAVs flies with a predefined plan. While the bigger UAVs acting as the actors and the sink follow a path with rather stable positions with respect to each other unless a change in the topology is required. The UAVs acting as the sensor nodes are mobile also with respect to the actors and the sink. Therefore a random mobility profile is created in the simulation platform such that the sensor nodes move randomly in a sphere with the sink at the center, while the sphere moves with the flight plan of the system. This profile simulates the movement of the nodes in the plume for data collection.

According to the optimization criteria, the efficiency of the proposed algorithm is studied using the following metrics: average, maximum and minimum weight values and cardinality of the actors.

\section{B. Simulation results}

1) Experiment 1: As explained in "System Model", each sensor node has its weight, which is a value between zero and the weight of the actor, $k$. The weight of a sensor node decreases by one with each hop it gets further from the actor. The collected information on a sensor node can be transmitted to an actor through the path of the sensor nodes with increasing weight values. Therefore, in contrast to many of the three dimensional positioning approaches in literature, the coverage of the three dimensional space is not the most critical criterion to measure the performance of our approach. The sensor measurements can be collected from a large volume of space by utilizing the weight attribute of the sensor nodes. Therefore we use another metric, average weight value, instead of coverage for the performance assessment of our protocol. Fig. 6 shows the average weight values of the sensor nodes in the simulations for all possible actor geometries.

Fig. 6 shows that the geometries formed by more actors result in higher average weight values in the network, which means less number of hops for the sensor nodes to transmit the collected information to the actors. The number of unconnected nodes is also decreasing as the geometries get larger. An interesting characteristic of the graph in Fig. 6 is the high difference in the average weight between trigonal planar geometry to tetrahedral geometry. Thus, it shows that the geometry gives better performance when more than one plane of actors are used.

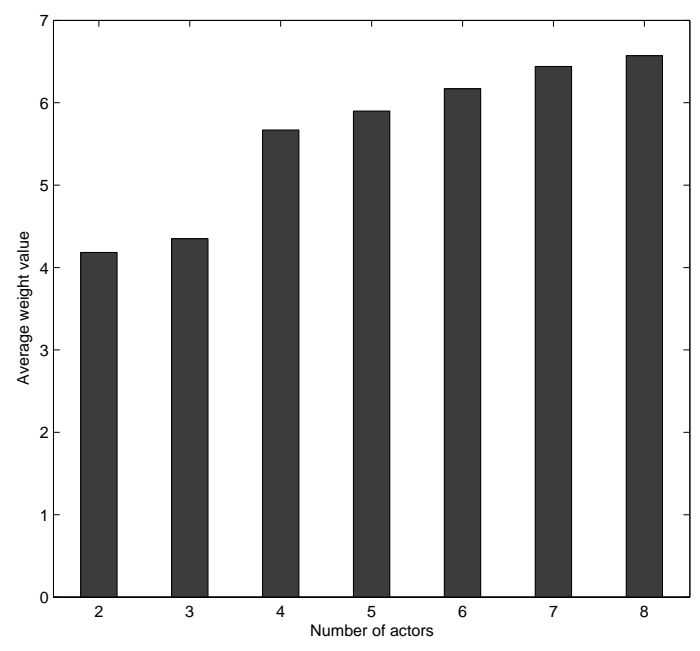

Figure 6. Average weight values for different geometries.

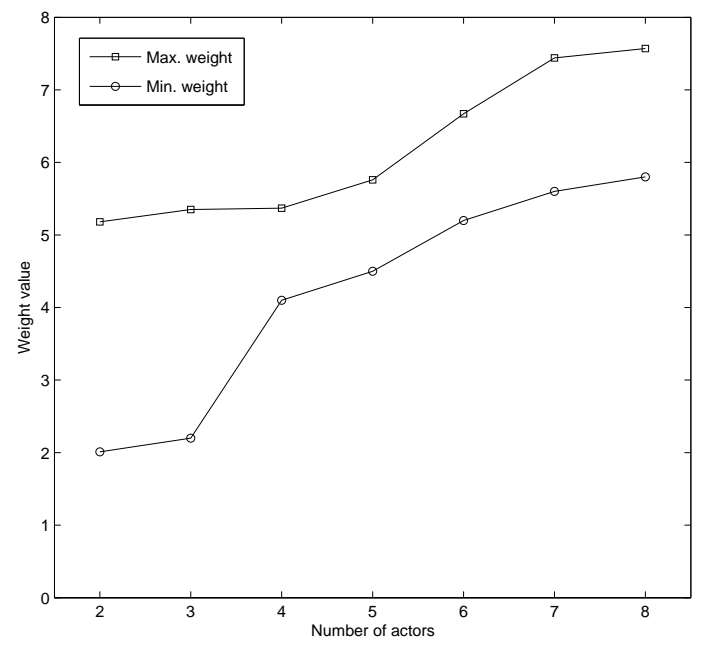

Figure 7. Maximum and minimum weight values for all geometries.

2) Experiment 2: Mobility is a fundamental characteristic of our application scenario. UAVs fly continuously with perturbations in their main flight paths. While the average weight value is critical, the maximum and minimum weight values are also important to assess the suitability of our positioning approach to the mobility of the nodes in our application scenario. The maximum and the minimum weight values averaged over the nodes for all possible geometries are given in Fig. 7.

Better positioning of actors denotes better sharing of the sensor nodes while they are moving. Hence if the average maximum weight value in the network is large even when the average weight is high, it indicates an ineffective sharing of the nodes as they move in the network. It can be observed in Fig. 7 that as the geometries evolve, the average minimum weight value increases and the difference between the minimum and the maximum weights decreases. Thus, the weights are distributed fairly in the network when the actors are positioned according to our positioning approach. This 


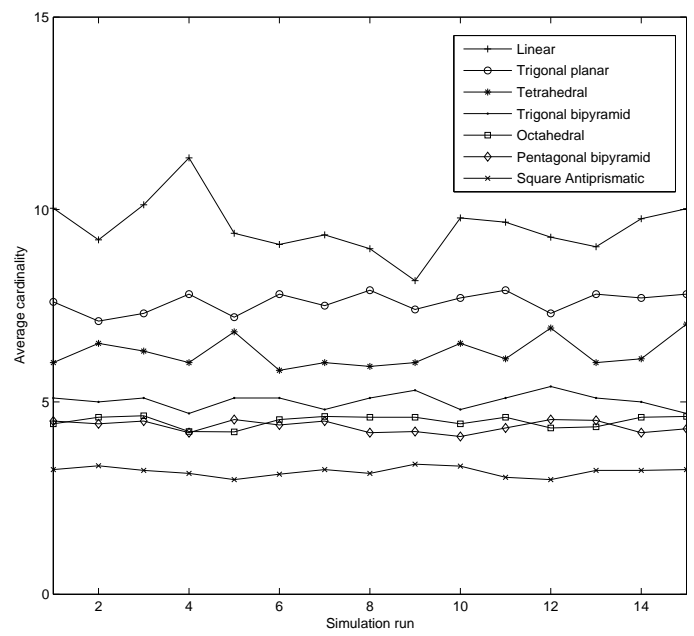

Figure 8. Cardinality of actors for different geometries.

result becomes important when combined with the results of the first simulation set, since it shows that the important factor in performance improvement is not only the increase in number of actors but also the geometries used.

3) Experiment 3: The cardinality of the actors is used as the metric in experiment 3 . While using multiple powerful UAVs as actors, the concurrency becomes essential for an effective utilization of the system. For this experiment, a single actor is chosen from each geometry and the fluctuation in the average cardinality of the actors in each simulation are shown in Fig. 8 for 15 simulation runs.

In the highly mobile and unstable topology of our scenario, the affiliations must be shared fairly among the actors so that the data loss and delay are minimized while the sensor nodes are transmitting the observed data to the actors. In Fig. 8, we observe that highest fluctuation occurs in the linear geometry and the fluctuation reduces as the number of actors in a geometry becomes larger. Therefore our approach is suitable for the highly unprecedented topology of the introduced application scenario.

\section{CONCLUSION}

In this paper, initial studies on a novel and scalable algorithm for positioning of actors in aerial WSANs is introduced. The goal of the proposed protocol, namely APAWSAN, is to improve the on-site monitoring of the plume in a volcanic eruption scenario. The particular scenario has its own challenges, which require robustness and high adaptability to failures. In order to overcome these challenges in a scalable way, all communication in the system is designed as locality preserving and the positioning algorithm remained lightweight by utilizing the properties of VSEPR theory geometries. We show that APAWSAN provides high connectivity and coverage for the sensor node network.

Some of the future work includes extending the actor positioning to large networks as well as further exploring the concepts of VSEPR theory and molecular geometry as applied to three dimensional positioning problem. For instance, the molecules, the ligands of which are not identical, can be used as a basis for the algorithms to position networks composed of nonhomogenous nodes.

\section{ACKNOWLEDGEMENT}

The authors would like to thank OPNET Technologies Inc. for supporting this research by providing OPNET Modeler software under OPNET University Program.

\section{REFERENCES}

[1] R. Verdone, D. Dardari, G. Mazzini, and A. Conti, Wireless Sensor and Actuator Networks: Technologies, Analysis and Design. Academic Press, 2008.

[2] S. M. N. Alam and Z. J. Haas, "Coverage and connectivity in threedimensional networks," in Proc. of the 12th annual international conference on Mobile computing and networking, ser. MobiCom '06, September 2006, pp. 346-357.

[3] V. Ravelomana, "Extremal properties of three-dimensional sensor networks with applications," IEEE Transactions on Mobile Computing, vol. 3, no. 3, pp. 246-257, July/September 2004.

[4] D. Pompili, T. Melodia, and I. F. Akyildiz, "Deployment analysis in underwater acoustic wireless sensor networks," in Workshop on Underwater Networks (WUWNET), September 2006, pp. 48-55.

[5] H. Zhou, S. Xia, M. Jin, and H. Wu, "Localized algorithm for precise boundary detection in 3D wireless networks," in Proc. of International Conference on Distributed Computing Systems, June 2010, pp. 744-753.

[6] X. Bai, C. Zhang, D. Xuan, and W. Jia, "Full-coverage and kconnectivity $(\mathrm{k}=14,6)$ three dimensional networks," in Proc. of IEEE INFOCOM, April 2009, pp. 388-396.

[7] P. I.-S. Chiang and W.-C. Peng, "Slab routing: Adapting twodimensional geographic routing to three-dimensions," in Proc. of IEEE SECON, July 2009, pp. 1-9.

[8] G. Astuti, G. Giudice, D. Longo, C. D. Melita, G. Muscato, and A. Orlando, "An overview of the "volcan Project": An UAS for Exploration of Volcanic Environments," J. Intell. Robotics Syst., vol. 54, no. 1-3, pp. 471-494, March 2009.

[9] A. Purohit and P. Zhang, "Sensorfly: A controlled-mobile aerial sensor network," in Proc. of the ACM Conference on Embedded Networked Sensor Systems (SenSys), 2009, pp. 327-328.

[10] J. Elston and E. Frew, "Hierarchical distributed control for search and tracking by heterogeneous aerial robot networks," in Proc. of IEEE International Conference on Robotics and Automation, May 2008, pp. 170-175.

[11] J. Elston, E. W. Frew, D. Lawrence, P. Gray, and B. Argrow, "Netcentric communication and control for a heterogeneous unmanned aircraft system," J. Intell. Robotics Syst., vol. 56, no. 1-2, pp. 199-232, September 2009.

[12] M. Dumiak, "Robocopters unite!" Spectrum, IEEE, vol. 46, no. 2, p. 12, 2009.

[13] J. Allred, A. B. Hasan, S. Panichsakul, W. Pisano, P. Gray, J. Huang, R. Han, D. Lawrence, and K. Mohseni, "SensorFlock: An airborne wireless sensor network of micro-air vehicles," in Proc. of the International Conference on Embedded Networked Sensor Systems (SenSys), November 2007, pp. 117-129.

[14] N. V. Sidgwick and H. M. Powell, "Bakerian lecture. stereochemical types and valency groups," Proc. of the Royal Society of London. Series A, Mathematical and Physical Sciences, vol. 176, no. 965, pp. 153-180, October 1940.

[15] R. Gillespie and R. Nyholm, "Inorganic stereochemistry," Quart. Rev. Chem. Soc., vol. 11, pp. 339-380, 1957.

[16] K. Valavanis, P. Oh, and L. Piegl, Unmanned Aircraft Systems: International Symposium on Unmanned Aerial Vehicles, UAV'08. Springer, 2008.

[17] M. I. Akbas, M. R. Brust, and D. Turgut, "SOFROP: Self-organizing and fair routing protocol for wireless networks with mobile sensors and stationary actors," in Proc. of 2010 Local Computer Networks (LCN), October 2010, pp. 456-463.

[18] "Opnet modeler," http://www.opnet.com. 